\title{
A Time-Based Meta-Analysis on the Incidence of New Onset Diabetes after Liver Transplantation
}

\author{
Yip Han Chin ${ }^{1}$, Hon Qin Marcus Tan ${ }^{1}$, Cheng Han Ng ${ }^{1} * \mathbb{\infty}$, Darren Jun Hao Tan ${ }^{1}$, Snow Yunni Lin ${ }^{1}$, \\ Daniel Q. Huang ${ }^{1,2,3 \mathbb{D}}$, Chin Meng Khoo ${ }^{1,2,+}$ and Mark Dhinesh Muthiah ${ }^{1,2,3, *,+}$ \\ 1 Yong Loo Lin School of Medicine, National University of Singapore, Singapore 117597, Singapore; \\ c.yiphan@u.nus.edu (Y.H.C.); e0360761@u.nus.edu (H.Q.M.T.); e0433903@u.nus.edu (D.J.H.T.); \\ e0416073@u.nus.edu (S.Y.L.); daniel_huang@nuhs.edu.sg (D.Q.H.); mdckcm@nus.edu.sg (C.M.K.) \\ 2 Department of Medicine, National University Hospital, Singapore 119074, Singapore \\ 3 National University Centre for Organ Transplantation, National University Hospital, \\ Singapore 119074, Singapore \\ * Correspondence: e0360747@u.nus.edu or chenhanng@gmail.com (C.H.N.); mdcmdm@nus.edu.sg (M.D.M.) \\ + These 2 authors supervised the work equally as senior authors.
}

Citation: Chin, Y.H.; Tan, H.Q.M.; Ng, C.H.; Tan, D.J.H.; Lin, S.Y.; Huang, D.Q.; Khoo, C.M.; Muthiah, M.D. A Time-Based Meta-Analysis on the Incidence of New Onset Diabetes after Liver Transplantation. J. Clin. Med. 2021, 10, 1045. https://doi.org/ $10.3390 /$ jcm10051045

Academic Editor: Michael Fuchs

Received: 20 January 2021

Accepted: 24 February 2021

Published: 3 March 2021

Publisher's Note: MDPI stays neutral with regard to jurisdictional claims in published maps and institutional affiliations.

Copyright: (c) 2021 by the authors. Licensee MDPI, Basel, Switzerland. This article is an open access article distributed under the terms and conditions of the Creative Commons Attribution (CC BY) license (https:/ / creativecommons.org/licenses/by/ $4.0 /)$.

\begin{abstract}
NODAT (new-onset diabetes after transplantation) is an important complication after liver transplant, however, there is variation in the reported incidence of NODAT. Therefore, a metaanalysis was performed to estimate the incidence of NODAT in liver transplant. Electronic databases were searched for articles regarding NODAT incidence after liver transplantation. Incidence of NODAT were analyzed at six different timepoints. Summary statistics were calculated using a generalized linear mixed model in random effects. 28 articles were included and out of a pooled population of 71,257 patients, overall incidence of NODAT was found to be $15.51 \%, 16.09 \%, 18.30 \%$, $20.86 \%, 18.08 \%, 25.05 \%$ for three-months, six-months, one-year, three-year, five-year, and ten-year timepoints respectively. After a sensitivity analysis which only included articles with clear definitions of NODAT, the incidence of NODAT was found to be higher at three-year (21.79\%), five-year (25.82\%), and ten-year (44.95\%) timepoints. Subgroup analysis according to ethnicity found no significant differences for all timepoints. However, studies with predominantly Asian participants generally had a higher incidence of NODAT. In conclusion, this meta-analysis provides a pooled estimate of the incidence of NODAT following liver transplantation. Further studies are required to provide a more comprehensive understanding on how ethnicity can affect the incidence of NODAT.
\end{abstract}

Keywords: NODAT; liver transplantation; new onset diabetes after transplantation; type 2 diabetes; incidence

\section{Introduction}

Liver transplantation is the only definitive treatment for end stage liver disease, and one of the only curative treatment options for hepatocellular carcinoma (HCC) [1]. Newonset diabetes after transplantation (NODAT) is a common and important complication estimated to occur in 15-30\% of recipients who were not known to be diabetic before the transplant [2]. It is also associated with an increased risk of early mortality [3], major cardiovascular events [4], renal impairment [5], biliary strictures [6], rejection episodes [7], and graft loss [8]. Despite its prevalence and important effects on patient outcomes, an international consensus guideline was only established in 2003 by the World Health Organization (WHO) following the American Diabetes Association (ADA) methods for diagnosing diabetes [9].

Risk factors for NODAT after liver transplant can generally be classified into nonmodifiable and modifiable factors [10]. Non-modifiable factors which increase the risk of NODAT include host factors such as older age [11], family history of DM, ethnicity [11], underlying diseases such as hepatitis $C$ virus $(\mathrm{HCV})$ infection and liver cirrhosis $[6,12,13]$, 
and donor factors such as donor age ( $>60$ years old) [14], presence of liver steatosis in donor livers [15], and male donors [6]. While modifiable factors which raise the risk of NODAT may include the type of immunosuppressive regimens utilised, such as corticosteroids or tacrolimus-containing regimens [11,12], high body mass index (BMI) [12] and length of intensive care unit (ICU) stay [6]. The effects of these in relations to genetics and ethnicity have also been brought into attention in recent years [16]. Hence, better identification of these risk factors and how it affects the incidence of NODAT in countries may prove crucial to improve the outcomes of liver transplant patients.

While the incidence and risk factors of NODAT in recipients of kidney transplants have been well established [17], the incidence of NODAT in liver transplant recipients is less clear, with several studies reporting heterogenous results due to differing definitions, length of follow-up and diagnostic criteria $[2,10,18]$. A previous meta-analysis has been conducted by Heisel et al. in 2003 [19]. However, this was before the international consensus by the WHO and ADA guidelines and this would have led to increased variations in the threshold for the diagnosis and reporting of NODAT cases in their included articles [19]. Thus, this meta-analysis was conducted to estimate the pooled incidence of NODAT after liver transplantation, adjusting for the different definitions of NODAT and analysing the incidence at predefined timepoints.

\section{Materials and Methods}

\subsection{Search Strategy}

Taking guidance from the Meta-Analysis of Observational Studies in Epidemiology (MOOSE) [20] and PRISMA [21], electronic databases (Medline and Embase) were searched for articles examining the incidence of NODAT after liver transplantation from inception till August 2020 and downloaded into EndnoteX9. Key search terms such as "incidence", "new-onset diabetes" and "liver transplantation" were used in the search strategy. Manual sieving of the references of the included articles was also conducted, and duplicate studies were removed using Endnote X9. The search was done in consultation with a medical librarian and the full search strategy can be found in the Supplementary Material 1.

\subsection{Inclusion Criteria and Data Extraction}

Two authors (YHC, HQMT) independently screened all articles retrieved from the search, and articles that met the inclusion criteria were marked for inclusion. Any discrepancies were resolved in consultation with an independent third author (CHN). Inclusion criteria included: (1) articles that studied the incidence of NODAT in adult patients after liver transplantation, (2) original articles that were translated into or written in the English language, (3) articles examining the incidence of NODAT at specific timepoints after liver transplantation, namely: three-months, six-months, one-year, three-year, five-year and ten-year timepoints. Exclusion criteria included: (1) articles that studied the incidence of NODAT following other forms of organ transplant such as kidney transplant, (2) articles that included pediatric populations, (3) articles that examined NODAT from the same database or hospital centers with overlapping time periods, and (4) dual organ recipients (heart and liver transplant, kidney and liver transplant, etc.).

Key data such as patient demographics (BMI, age and gender), characteristics of included articles (sample size, country, follow-up time), definition of NODAT (WHO and ADA), postoperative medications (tacrolimus, steroids) and incidence of NODAT at the intervals previously mentioned were extracted by two authors (YHC, HQMT) independently into a predefined datasheet. Any discrepancy was resolved by discussion with a third author (CHN). Manual extraction of the incidence of NODAT from the Kaplan-Meier curves using WebPlotDigitalizer was used when raw numbers were not available from the articles. Estimated values of the mean and standard deviation were derived through formulas when they were not provided [22]. 


\subsection{Statistical Analysis and Quality Assessment}

All analyses were conducted using R (RStudio 1.3.1073). Pooled proportions of the incidence of NODAT after liver transplantation were analyzed at the intervals previously mentioned. Analysis of proportions was undertaken using a generalized linear mixed model (GLMM) instead of Freeman-Tukey double arcsine transformation as GLMM has been shown to be the most accurate method for transformation for meta-analysis of single proportions in numerous simulation studies [23]. No continuity correction was applied and all analyses were conducted in random effects regardless of heterogenicity measures $\left(\mathrm{I}^{2}\right.$, tau, Cochran $Q$ test) [23]. As single arm meta-analysis data are usually heterogenous, singlearm prevalence reviews often show substantial heterogeneity when the interpretation of $\mathrm{I}^{2}$ alone is used in the quantification of heterogeneity $[24,25] . \mathrm{I}^{2}$ can be especially misleading in large datasets as $\mathrm{I}^{2}$ increases with sample size [26].

A subsequent sensitivity analysis was conducted to include only articles using WHO and ADA criteria for NODAT for a more homogenous analysis. In addition, further subgroup analysis was also conducted based on the predominant ethnicity of the patients in the included study, where articles were classified into articles with predominantly Asian patients and articles with predominantly Western patients [27,28]. Analysis of publication bias was also conducted through visual inspection of funnel plots when sufficient studies were available $(n>9)$ [29]. Quality assessment of cohort studies was conducted via the Newcastle Ottawa Scale (NOS), which evaluates the quality of the articles using three criteria including selection, comparability and outcome [30].

\section{Results}

\subsection{Search Results and Study Characteristics}

1372. articles were identified after the search, and 170 articles were selected for full text review, of which 28 met the inclusion criteria. Figure 1 shows the flowchart of the inclusion process. The articles originated from various countries, with six articles originating from each USA [31-36] and China [6,15,37-39] respectively, three from France [40-42], two each from India [43,44], Japan [45,46], Spain [47,48], one each from Canada [49], Egypt [50], Germany [51], Iran [52], Korea [53], Taiwan [54], UK [55]. Studies were conducted from 1989 till 2018. A total of 71,257 patients were included in the various studies and the mean age of the participants ranged from 39.24 to 55.61 years old. Quality assessment of included articles was conducted, and most articles were of moderate to high quality. The details of the quality assessment and characteristics of the included articles are presented in Table 1 , and articles included in this article can be seen in the Supplementary References list.

\subsection{Definition of NODAT}

Definition of NODAT was based on the $2003 \mathrm{WHO}$ and ADA criteria [9], defined as a fasting glucose level of $\geq 7.0 \mathrm{mmol} / \mathrm{L}$, or a non-fasting glucose level of $\geq 11.1 \mathrm{mmol} / \mathrm{L}$ confirmed on at least two occasions or a need for antidiabetic drugs after the first posttransplant month [9]. Nine articles defined NODAT without reference to the WHO and ADA criteria, and 19 articles followed the WHO and ADA guidelines for NODAT after liver transplantation.

\subsection{Overall Incidence of NODAT}

The incidence of NODAT after liver transplantation was analyzed at three-months, six-months, one-year, three-year, five-year and ten-year timepoints. The overall incidence of NODAT after liver transplantation was found to range between $15 \%$ to $25 \%$ (Table 2). The pooled incidence of NODAT was $15.5 \%$ (CI: $11.5 \%$ to $20.7 \%$, Figure 2), $16.1 \%$ (CI: $10.9 \%$ to $23.1 \%$, Figure 2), $18.3 \%$ (CI: $14.8 \%$ to $22.4 \%$, Figure 3), $20.9 \%$ (CI:13.0\% to $31.8 \%$ ), $18.08 \%$ (CI:10.3\% to $29.9 \%$ ), $25.05 \%$ (CI: $11.2 \%$ to $47.1 \%$ ) for three-months, six-months, one-year, three-year, five-year and ten-year timepoints respectively (Table 2). Visual inspection of funnel plot suggests significant publication bias for 1-year (Figure 4), 6-months, three-years, and five-years timepoints (Supplementary Material). 


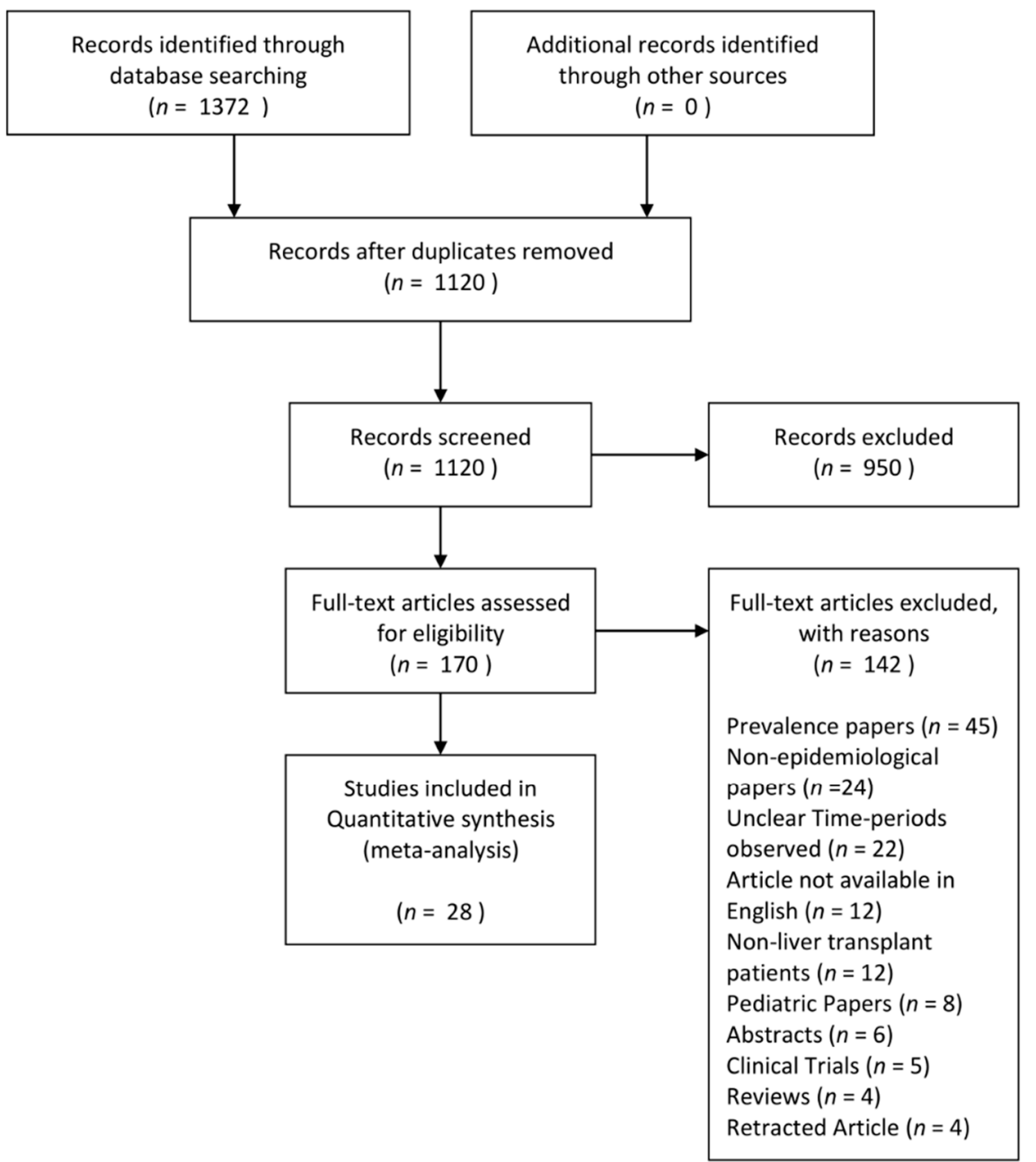

Figure 1. Flow chart of systematic literature search and screening for incidence of New onset diabetes after liver transplantation.

Table 1. Summary of Included Articles.

\begin{tabular}{|c|c|c|c|c|c|c|c|}
\hline Author & Year & $\begin{array}{c}\text { Sample } \\
\text { Size }\end{array}$ & $\begin{array}{l}\text { Gender } \\
\text { (M) }\end{array}$ & Age & BMI & $\begin{array}{c}\text { Immunosuppressive } \\
\text { Regimen }\end{array}$ & $\begin{array}{l}\text { NOS } \\
\text { Score }\end{array}$ \\
\hline Jain AB et al. [31] & 1999 & 121 & 0.56 & $46.3 \pm 12.3$ & - & $\begin{array}{l}\text { Tacrolimus, Cyclosporine, } \\
\text { Prednisone, Azathioprine }\end{array}$ & 4 \\
\hline Driscoll et al. [32] & 2006 & 115 & 0.71 & $48.5 \pm 10.9$ & $27.4 \pm 6.1$ & $\begin{array}{l}\text { Tacrolimus, Cyclosporine, } \\
\text { Sirolimus, MMF, } \\
\text { Azathioprine }\end{array}$ & 6 \\
\hline Saliba et al. [42] & 2007 & 211 & 0.71 & $52.7 \pm 9.8$ & $25.3 \pm 4.68$ & Tacrolimus, Steroids & 6 \\
\hline Oufroukhi et al. [41] & 2008 & 141 & 0.67 & $52.6 \pm 10$ & $24.7 \pm 5$ & Tacrolimus, Steroids, MMF & 5 \\
\hline Zhao et al. [38] & 2009 & 84 & 0.83 & $42.5 \pm 9.1$ & $22.2 \pm 3.2$ & Tacrolimus, Cyclosporine & 4 \\
\hline Carey et al. [36] & 2011 & 225 & 0.71 & $51.7 \pm 9.8$ & $28.1 \pm 5.2$ & $\begin{array}{l}\text { Tacrolimus, Cyclosporine, } \\
\text { Sirolimus }\end{array}$ & 6 \\
\hline Honda et al. [45] & 2013 & 161 & 0.47 & $47.2 \pm 12.9$ & $22.6 \pm 3.8$ & $\begin{array}{l}\text { Tacrolimus, Cyclosporine, } \\
\text { Steroids, MMF }\end{array}$ & 5 \\
\hline Rubin et al. [48] & 2013 & 158 & 0.67 & $44.75 \pm 9.60$ & $25.25 \pm 3.39$ & $\begin{array}{l}\text { Cyclosporine, Tacrolimus, } \\
\text { Azathioprine, Steroids }\end{array}$ & 6 \\
\hline Cho et al. [53] & 2014 & 364 & 0.69 & $49.98 \pm 9.18$ & $23.62 \pm 3.23$ & Steroids & 6 \\
\hline Parvizi et al. [52] & 2014 & 350 & 0.58 & $39.24 \pm 16.24$ & $22.70 \pm 4.63$ & $\begin{array}{c}\text { Tacrolimus, Mycophenolate, } \\
\text { Prednisone }\end{array}$ & 4 \\
\hline $\begin{array}{l}\text { Varghese et al. [44] } \\
\text { Younossi et al. [34] }\end{array}$ & $\begin{array}{l}2014 \\
2014\end{array}$ & $\begin{array}{c}32 \\
18571\end{array}$ & $\begin{array}{l}0.90 \\
0.75\end{array}$ & $\begin{array}{c}44.3 \pm 12.4 \\
54.04+7.37\end{array}$ & $2798+545$ & $\begin{array}{c}\text { Tacrolimus, MMF } \\
\text { Tacrolimus. MMF Steroids }\end{array}$ & $\frac{4}{5}$ \\
\hline Cuervas-Mons et al. & 2015 & 117 & 0.79 & $55.60 \pm 8.31$ & $27.77 \pm 4.48$ & Tacrolimus & 4 \\
\hline $\begin{array}{l}\text { Gebhardt et al. [51] } \\
\text { Hartog et al. [55] }\end{array}$ & $\begin{array}{l}2015 \\
2015\end{array}$ & $\begin{array}{c}81 \\
430\end{array}$ & $\begin{array}{c}0.70 \\
0.8\end{array}$ & $\begin{array}{c}55.10 \pm 10.67 \\
48.25 \pm 9.23\end{array}$ & $\begin{aligned} 28.92 & \pm 6.05 \\
26.4 & \pm 5.1\end{aligned}$ & $\begin{array}{l}\text { Tacrolimus, Prednisone } \\
\text { Tacrolimus, Steroids }\end{array}$ & $\begin{array}{l}4 \\
4\end{array}$ \\
\hline
\end{tabular}


Table 1. Cont.

\begin{tabular}{|c|c|c|c|c|c|c|c|}
\hline Author & Year & $\begin{array}{l}\text { Sample } \\
\text { Size }\end{array}$ & $\begin{array}{l}\text { Gender } \\
\text { (M) }\end{array}$ & Age & BMI & $\begin{array}{c}\text { Immunosuppressive } \\
\text { Regimen }\end{array}$ & $\begin{array}{l}\text { NOS } \\
\text { Score }\end{array}$ \\
\hline Stepanova et al. [35] & 2015 & 17,184 & 0.59 & $51.82 \pm 12.54$ & $27.27 \pm 5.87$ & Tacrolimus, Steroids, MMF & 6 \\
\hline Liu et al. [54] & 2016 & 2248 & 0.69 & $43.95 \pm 19.14$ & - & $\begin{array}{c}\text { Tacrolimus, Cyclosporine, } \\
\text { MMF, Rapamune, } \\
\text { Everolimus }\end{array}$ & 5 \\
\hline Li et al. [13] & 2016 & 18,741 & 0.67 & $53.62 \pm 10.36$ & $28.11 \pm 5.79$ & Tacrolimus, Steroids, MMF & 6 \\
\hline Ling et al. [6] & 2016 & 10,204 & 0.83 & $48.20 \pm 10.01$ & $22.79 \pm 2.91$ & $\begin{array}{l}\text { Tacrolimus, Cyclosporine, } \\
\text { Corticosteroids }\end{array}$ & 5 \\
\hline Saliba et al. [40] & 2016 & 180 & 0.81 & $54.25 \pm 8.42$ & - & MMF & 6 \\
\hline Song et al. [37] & 2016 & 528 & 0.85 & $44.93 \pm 9.41$ & - & $\begin{array}{c}\text { Tacrolimus, Corticosteroids, } \\
\text { MMF }\end{array}$ & 5 \\
\hline $\begin{array}{l}\text { Yagi et al. [46] } \\
\text { Cen et al. [39] }\end{array}$ & $\begin{array}{l}2016 \\
2017\end{array}$ & $\begin{array}{l}175 \\
256\end{array}$ & $\begin{array}{l}0.50 \\
0.84\end{array}$ & $\begin{array}{c}51 \pm 11 \\
47.92 \pm 7.34\end{array}$ & $\begin{aligned} 23.8 & \pm 0.3 \\
22.53 & \pm 3.02\end{aligned}$ & $\begin{array}{c}\text { Tacrolimus, MMF } \\
\text { Tacrolimus, MMF, Steroids }\end{array}$ & $\begin{array}{l}5 \\
4\end{array}$ \\
\hline Xue et al. [15] & 2017 & 763 & 0.85 & $48.78 \pm 10.23$ & $23.06 \pm 2.4$ & $\begin{array}{l}\text { Tacrolimus, MMF, } \\
\text { Corticosteroids }\end{array}$ & 4 \\
\hline Lieber et al. [33] & 2019 & 415 & 0.68 & 54.38 & 28.87 & $\begin{array}{l}\text { Tacrolimus, Mycophenolate, } \\
\text { Sirolimus }\end{array}$ & 5 \\
\hline Aravinthan et al. [49] & 2019 & 2209 & 0.67 & $53.67 \pm 9.64$ & $26.67 \pm 5.19$ & $\begin{array}{l}\text { Tacrolimus, Cyclosporine, } \\
\text { Sirolimus, Prednisone, } \\
\text { Mycophenolate }\end{array}$ & 6 \\
\hline Oommen et al. [43] & 2020 & 51 & - & $45.6 \pm 9.6$ & $24.52 \pm 4.63$ & $\begin{array}{c}\text { Tacrolimus, Glucocorticoids, } \\
\text { Azathioprine, } \\
\text { Mycophenolate }\end{array}$ & 4 \\
\hline Hashim et al. [50] & 2020 & 100 & 0.91 & $52 \pm 7.7$ & $27.2 \pm 4.4$ & $\begin{array}{c}\text { Tacrolimus, Cyclosporine, } \\
\text { mTOR, Steroids }\end{array}$ & 5 \\
\hline
\end{tabular}

"_"-Information was not available in the articles; All numbers are presented in mean \pm standard deviation unless stated otherwise. BMI - Body Mass Index, DM-Diabetes Mellitus, M - Male, MMF-Mycophenolate Mofetil, mTOR—mammalian target of rapamycin, NOS-Newcastle Ottowa Scale.

Table 2. Incidence of New-Onset Diabetes after Transplantation (NODAT) after Liver Transplantation.

\begin{tabular}{|c|c|c|c|c|c|c|c|c|}
\hline $\begin{array}{l}\text { Study } \\
\text { Period }\end{array}$ & $\begin{array}{l}\text { Total } \\
\text { Papers }\end{array}$ & $\begin{array}{l}\text { Total } \\
\text { Sample } \\
\text { Size }\end{array}$ & $\begin{array}{c}\text { Pooled } \\
\text { Incidence }\end{array}$ & $\begin{array}{c}\text { Total } \\
\text { Sample } \\
\text { Size (after } \\
\text { Sensitivity } \\
\text { Analysis) }\end{array}$ & $\begin{array}{l}\text { Pooled } \\
\text { Incidence }\end{array}$ & $\begin{array}{l}\text { Incidence of } \\
\text { NODAT from } \\
\text { Studies from } \\
\text { Predominantly } \\
\text { Western } \\
\text { Populations }\end{array}$ & $\begin{array}{c}\text { Incidence of } \\
\text { NODAT from } \\
\text { Studies from } \\
\text { Predominantly } \\
\text { Asian } \\
\text { Populations }\end{array}$ & $p$-Value \\
\hline 3 months & 7 & 11226 & $\begin{array}{c}15.51 \% \\
\text { (CI: } 11.47 \% \\
\text { to } 20.65 \% \text { ) }\end{array}$ & 11367 & $\begin{array}{c}14.36 \% \\
\text { (CI: } 11.09 \% \\
\text { to } 18.38 \%)\end{array}$ & $\begin{array}{c}14.65 \% \\
\text { (CI: } 10.81 \% \text { to } \\
19.54 \%)\end{array}$ & $\begin{array}{c}13.97 \% \text { (CI: } 9.65 \% \\
\text { to } 19.81 \%)\end{array}$ & 0.8424 \\
\hline 6 months & 10 & 46203 & $\begin{array}{c}16.09 \% \\
\text { (CI: } 10.89 \% \\
\text { to } 23.12 \%)\end{array}$ & 11291 & $\begin{array}{l}17.12 \% \\
\text { (CI:12.68\% } \\
\text { to } 22.70 \%)\end{array}$ & $\begin{array}{c}15.87 \% \\
\text { (CI:10.07\% to } \\
24.11 \%)\end{array}$ & $\begin{array}{c}17.83 \% \\
\text { (CI: } 12.08 \% \text { to } \\
25.51 \% \text { ) }\end{array}$ & 0.6926 \\
\hline 1 year & 24 & 65845 & $\begin{array}{c}18.30 \% \\
\text { (CI: } 14.78 \% \\
\text { to } 22.44 \%)\end{array}$ & 64298 & $\begin{array}{c}19.22 \% \\
\text { (CI:15.30\% } \\
\text { to } 23.88 \%)\end{array}$ & $\begin{array}{c}16.39 \% \\
\text { (CI:12.83\% to } \\
20.29 \%)\end{array}$ & $\begin{array}{c}20.04 \% \\
(\mathrm{CI}: 15.51 \% \text { to } \\
25.00 \%)\end{array}$ & 0.2318 \\
\hline 3 years & 10 & 40882 & $\begin{array}{c}20.86 \% \\
\text { (CI: } 12.95 \% \\
\text { to } 31.84 \%)\end{array}$ & 20711 & $\begin{array}{c}21.79 \% \\
\text { (CI:11.19\% } \\
\text { to } 38.12 \%)\end{array}$ & $\begin{array}{l}14.19 \%(\mathrm{CI}: 1.62 \% \\
\text { to } 62.46 \%)\end{array}$ & $\begin{array}{c}26.35 \% \\
\text { (CI:18.44\% to } \\
36.16 \%)\end{array}$ & 0.5204 \\
\hline 5 years & 10 & 35680 & $\begin{array}{c}18.08 \% \\
\text { (CI:10.25\% } \\
\text { to } 29.90 \% \text { ) } \\
25.05 \%\end{array}$ & 20318 & $\begin{array}{c}25.82 \% \\
\text { (CI: } 10.90 \% \\
\text { to } 49.73 \%) \\
44.95 \%\end{array}$ & $\begin{array}{c}\text { 20.77\% (CI: } 2.88 \% \\
\text { to } 69.87 \%)\end{array}$ & $\begin{array}{c}30.94 \% \\
\text { (CI:16.61\% to } \\
50.20 \%)\end{array}$ & 0.6516 \\
\hline 10 years & 4 & 3697 & $\begin{array}{l}\text { (CI: } 11.17 \% \\
\text { to } 47.05 \%)\end{array}$ & 1291 & $\begin{array}{l}\text { (CI:30.56\% } \\
\text { to } 60.24 \%)\end{array}$ & - & - & - \\
\hline
\end{tabular}




\begin{tabular}{|c|c|c|c|c|c|}
\hline Study & Events & Total & NODAT & Events & $95 \%-\mathrm{Cl}$ \\
\hline \multicolumn{6}{|l|}{$3 \mathrm{~m}$} \\
\hline Driscoll et al 2006 & 28 & 115 & : & 24.35 & {$[16.83 ; 33.23]$} \\
\hline Saliba et al 2007 & 39 & 211 & $\div$ & 18.48 & {$[13.49 ; 24.39]$} \\
\hline Zhao et al 2009 & 7 & 74 & +4 & 9.46 & {$[3.89 ; 18.52]$} \\
\hline Ling et al 2016 & 1990 & 10204 & $\vdots+$ & 19.50 & {$[18.74 ; 20.28]$} \\
\hline Hartog et al 2015 & 52 & 430 & Fi 1 & 12.09 & {$[9.16 ; 15.55]$} \\
\hline Honda et al 2013 & 12 & 160 & $+\vdots 1$ & 7.50 & {$[3.94 ; 12.73]$} \\
\hline Varghese et al 2014 & 8 & 32 & $\frac{1}{1}$ & 25.00 & {$[11.46 ; 43.40]$} \\
\hline Fixed effect model & & 11226 & $\infty$ & 19.03 & {$[18.31 ; 19.76]$} \\
\hline \multirow{2}{*}{\multicolumn{3}{|c|}{$\begin{array}{l}\text { Random effects model } \\
\text { Heterogeneity: } I^{2}=86 \%, \tau^{2}=0.1625\end{array}$}} & $\ddot{\phi}$ & 15.51 & {$[11.47 ; 20.64]$} \\
\hline & & & & & \\
\hline \multicolumn{6}{|l|}{$6 \mathrm{~m}$} \\
\hline Driscoll et al 2006 & 26 & 115 & $1+$ & 22.61 & {$[15.33 ; 31.35]$} \\
\hline Saliba et al 2007 & 46 & 211 & + & 21.80 & {$[16.43 ; 27.99]$} \\
\hline Oufroukhi et al 2008 & 48 & 117 & $\longrightarrow$ & 41.03 & {$[32.02 ; 50.50]$} \\
\hline Ling et al 2016 & 2173 & 10204 & $\vdots$ & 21.30 & {$[20.50 ; 22.10]$} \\
\hline Hartog et al 2015 & 50 & 430 & +1 & 11.63 & {$[8.75 ; 15.04]$} \\
\hline Honda et al 2013 & 15 & 158 & $\mp$ & 9.49 & {$[5.41 ; 15.17]$} \\
\hline Liu et al 2016 & 96 & 2248 & 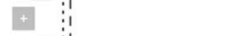 & 4.27 & {$[3.47 ; 5.19]$} \\
\hline Stepanova et al 2015 & 2236 & 17184 & $\therefore 1$ & 13.01 & {$[12.51 ; 13.52]$} \\
\hline Varghese et al 2014 & 5 & 32 & 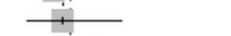 & 15.62 & [ $5.28 ; 32.79]$ \\
\hline Younossi et al 2014 & 3440 & 15504 & $1+$ & 22.19 & {$[21.54 ; 22.85]$} \\
\hline Fixed effect model & & 46203 & 1 & 17.61 & {$[17.26 ; 17.96]$} \\
\hline \multicolumn{3}{|c|}{ Random effects model } & $\stackrel{1}{i}$ & 16.09 & {$[10.89 ; 23.12]$} \\
\hline \multicolumn{3}{|c|}{ Heterogeneity: $I^{2}=100 \%, \tau^{2}=0.4880$} & & & \\
\hline \multirow{3}{*}{\multicolumn{2}{|c|}{$\begin{array}{l}\text { Fixed effect model } \\
\text { Random effects model }\end{array}$}} & 57429 & $\vdots$ & \multicolumn{2}{|c|}{$17.88[17.57 ; 18.20]$} \\
\hline & & & $\dot{0}$ & 15.80 & {$[12.10 ; 20.36]$} \\
\hline & 37 & & 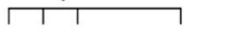 & & \\
\hline Residual heterogeneity: $I^{2}$ & $=98 \%$ & & 01020 & & \\
\hline
\end{tabular}

Figure 2. Incidence of New-Onset Diabetes after Transplantation (NODAT) at 3-months and 6-months after Liver Transplantation. $\mathrm{CI}=$ Confidence interval.

\begin{tabular}{|c|c|c|c|c|c|}
\hline Study & Events & Total & NODAT & Events & $95 \%-\mathrm{Cl}$ \\
\hline $12 \mathrm{~m}$ & & & & & \\
\hline Jain AB et al 1999 & 9 & 106 & +1 & 8.49 & {$[3.96 ; 15.51]$} \\
\hline Driscoll et al 2006 & 22 & 115 & 1 & 19.13 & {$[12.39 ; 27.52]$} \\
\hline Oufroukhi et al 2008 & 42 & 117 & 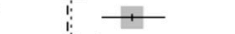 & 35.90 & {$[27.24 ; 45.29]$} \\
\hline Ling et al 2016 & 2337 & 10204 & + & 22.90 & {$[22.09 ; 23.73]$} \\
\hline Cho et al 2014 & 50 & 304 & + & 16.45 & {$[12.46 ; 21.10]$} \\
\hline Cuervas-Mons et al 2015 & 23 & 89 & & 25.84 & {$[17.14 ; 36.21]$} \\
\hline Gebhardt et al 2015 & 4 & 43 & $+i$ & 9.30 & [ $2.59 ; 22.14]$ \\
\hline Hartog et al 2015 & 41 & 430 & + & 9.53 & [ $6.93 ; 12.71]$ \\
\hline Hashim et al 2020 & 25 & 63 & 1 & 39.68 & {$[27.57 ; 52.80]$} \\
\hline Honda et al 2013 & 13 & 124 & + & 10.48 & {$[5.70 ; 17.26]$} \\
\hline Li et al 2016 & 2436 & 18741 & +1 & 13.00 & {$[12.52 ; 13.49]$} \\
\hline Lieber et al 2019 & 105 & 303 & + & 34.65 & {$[29.30 ; 40.31]$} \\
\hline Liu et al 2016 & 121 & 2248 & +1 & 5.38 & {$[4.49 ; 6.40]$} \\
\hline Oommen et al 2020 & 10 & 26 & + & 38.46 & {$[20.23 ; 59.43]$} \\
\hline Parvizi et al 2014 & 60 & 350 & $\frac{1}{1}$ & 17.14 & {$[13.34 ; 21.51]$} \\
\hline Rubin et al 2013 & 14 & 158 & +1 & 8.86 & {$[4.93 ; 14.42]$} \\
\hline Saliba et al 2016 & 48 & 180 & + & 26.67 & {$[20.36 ; 33.76]$} \\
\hline Song et al 2016 & 80 & 528 & + & 15.15 & {$[12.20 ; 18.50]$} \\
\hline Stepanova et al 2015 & 2820 & 14128 & $\div$ & 19.96 & {$[19.30 ; 20.63]$} \\
\hline Xue et al 2017 & 252 & 763 & + & 33.03 & {$[29.70 ; 36.49]$} \\
\hline Yagi et al 2016 & 30 & 115 & & 26.09 & {$[18.34 ; 35.10]$} \\
\hline Younossi et al 2014 & 2469 & 14574 & $i$ & 16.94 & {$[16.34 ; 17.56]$} \\
\hline Carey et al 2011 & 39 & 225 & $\because$ & 17.33 & {$[12.63 ; 22.92]$} \\
\hline Aravinthan et al 2019 & 314 & 1911 & + & 16.43 & {$[14.80 ; 18.17]$} \\
\hline Fixed effect model & & 65845 & 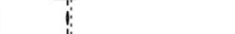 & 17.26 & {$[16.97 ; 17.55]$} \\
\hline \multicolumn{3}{|c|}{ Heterogeneity: $I^{2}=99 \%, \tau^{2}=0.3689$} & $\overbrace{i}^{1}$ & 18.30 & {$[14.77 ; 22.44]$} \\
\hline \multirow{2}{*}{\multicolumn{2}{|c|}{$\begin{array}{l}\text { Fixed effect model } \\
\text { Random effects model }\end{array}$}} & \multirow[t]{2}{*}{65845} & 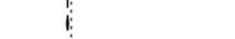 & \multirow{3}{*}{\multicolumn{2}{|c|}{$\begin{array}{l}17.26[16.97 ; 17.55] \\
18.30[14.77 ; 22.44]\end{array}$}} \\
\hline & & & $\dot{b}$ & & \\
\hline \multirow{2}{*}{\multicolumn{2}{|c|}{$\begin{array}{l}\text { Heterogeneity: } I^{2}=99 \%, \tau^{2}=0.36 \\
\text { Residual heterogeneity: } I^{2}=98 \%\end{array}$}} & & $\Gamma \mid$ & & \\
\hline & & & 01020 & & \\
\hline
\end{tabular}

Figure 3. Incidence of New-Onset Diabetes after Transplantation (NODAT) at 1-year after Liver Transplantation. $\mathrm{CI}=$ Confidence interval. 


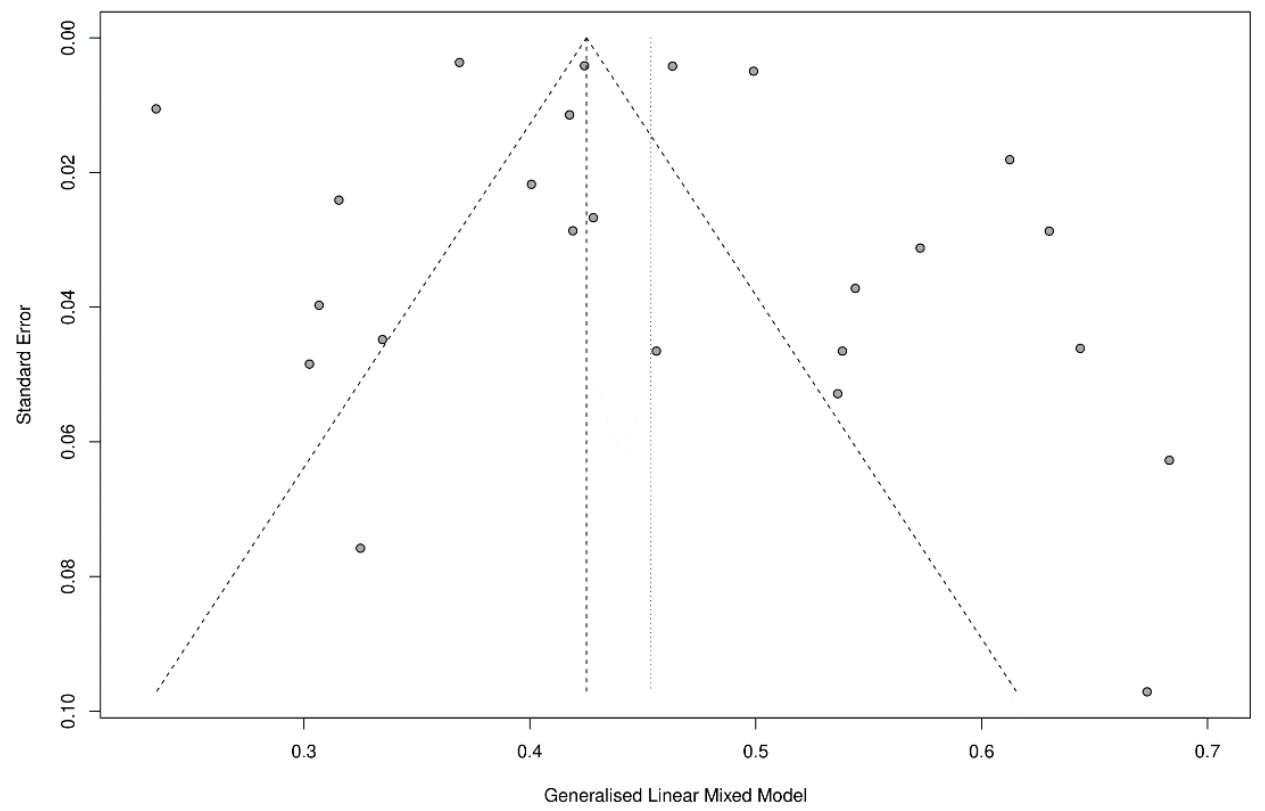

Figure 4. Funnel Plot of Incidence of New-Onset Diabetes after Transplantation (NODAT) at 1-year after Liver Transplantation.

A sensitivity analysis was conducted where only articles using the WHO and ADA definitions of NODAT were included in the analysis (Figure 5). The incidence of NODAT was relatively similar at three-months $(14.4 \%$; CI: $11.1 \%$ to $18.4 \%)$, six-months $(17.1 \%$; CI: $12.7 \%$ to $22.7 \%$ ), one-year (19.2\%; CI: $15.3 \%$ to $23.9 \%)$, but higher at three-year $(21.8 \%$; CI: $11.2 \%$ to $38.1 \%$ ), five-year ( $25.8 \%$; CI: $10.9 \%$ to $49.7 \%$ ), and ten-year $(45.0 \%$; CI:30.6\% to $60.2 \%$ ) timepoints when compared to overall analysis (Table 2).

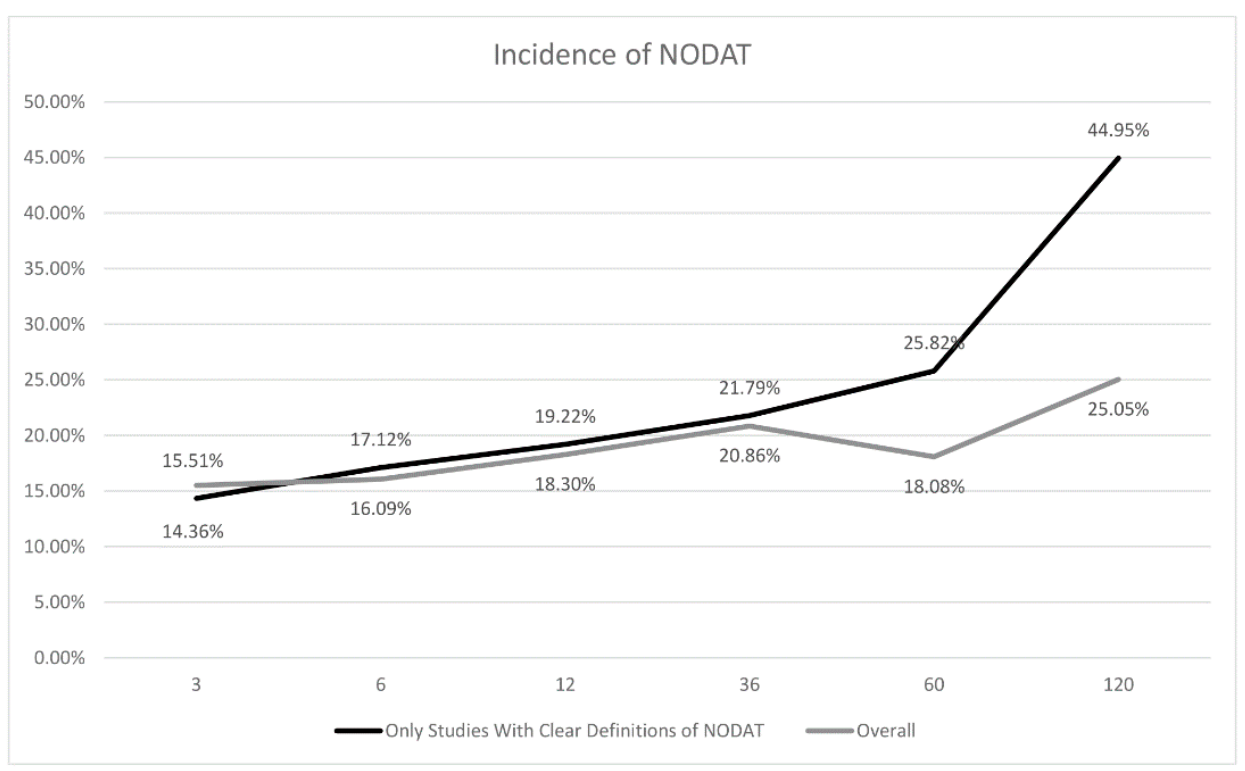

Figure 5. Incidence of New-Onset Diabetes after Transplantation (NODAT) at Various Timepoints.

After the sensitivity analysis, included articles were then subgrouped according to the predominant ethnicity of the patients and analyzed. No significant differences in the incidence of NODAT was found for all timepoints (Table 2). However, other than the threemonths timepoint, studies from predominantly Asian populations had a higher incidence of NODAT as compared to studies from predominantly Western populations for all other timepoints (Table 2). 


\section{Discussion}

NODAT has been observed to have an adverse effect on patient survival and graft survival and an increased incidence of infectious complications, where patients with NODAT had higher rates of postoperative bacterial infections and lower survival rates compared to patients without NODAT [5]. In addition, patients with NODAT are more likely to experience an acute rejection episode $[7,56]$. The results of this meta-analysis suggested that the overall incidence of NODAT after liver transplant at three-months, six-months, one-year, three-year, five-year, and ten-year timepoints ranged between $15 \%$ to $25 \%$. When considering only articles which had clear definitions of NODAT, the incidence of NODAT increased, and it ranged between $15 \%$ to $45 \%$. The incidence was comparable, if not higher than that of NODAT after renal transplant which ranged between $4 \%$ to $25 \%$ [12]. It is also higher in comparison to a previous meta-analysis conducted by Heisel et al., which ranged between $7.7 \%$ to $18.2 \%$ [19].

Evidence for the effect of ethnicity on the incidence of NODAT has been conflicting $[10,11,14,36]$. This meta-analysis found that studies with patients predominantly from Asian populations had a non-significant increase in incidence of NODAT compared to articles with patients predominantly from Western populations (Table 2). Previous studies have suggested that ethnicity may potentially affect the incidence of NODAT [10]. However, the influence of ethnicity on NODAT has yet to be confirmed [10], and these studies had small sample sizes in their analysis. Furthermore, there was high heterogeneity across the included studies, and current evidence on the issue is insufficient. More multicentric studies and trials would be needed to assess whether ethnicity could affect incidence of NODAT [16], and further studies aimed at explaining the sources of heterogeneity between the studies should be conducted.

In the overall analysis, incidence of NODAT at five-year was noted to have a decrease in the incidence compared to three-year incidence (Figure 5) likely due to the result of variable definitions. However, after sensitivity analysis for articles using WHO/ADA guidelines [9], a more temporal relationship with estimates of $25.82 \%$ and $44.95 \%$ for fiveyear and ten-year timepoints respectively was found. Our analysis also found that the incidence of NODAT was much higher in the three-year, five-year, and ten-year timepoints as compared to those one-year or less after the sensitivity analysis (Figure 5). This could be attributed to the lack of clear definitions of NODAT, and it has been an issue for the study of this condition $[9,10,12]$, leading to a variation in reported incidence of this condition. Additionally, a short observational period for NODAT ( $<1$ year) can also lead to an underestimation of the true incidence of NODAT [9], and many patients developed the condition many years after transplant $[9,10,12]$. Thus, reporting of incidence of NODAT should include timepoints longer than one-year to better understand the true incidence of NODAT.

While the importance of reporting the incidence of NODAT after one-year is important, we do note that the development of NODAT at five- and ten-years post-transplant may be significantly different compared to those at six-month or one-year post-transplant. Those developed at a later timing may be affected more by lifestyle habits such as diet, smoking and physical activity as well as the development of other comorbidities such as hyperlipidaemia and hypertension [57]. In addition, our analysis may be affected by survival bias, where patients who survive to ten-year posttransplant may be patients with lower BMI, of more benign disease and of a younger age, factors which can affect the development of NODAT (Supplementary Material 2) [58].

In addition, well known risk factors such as the use of living donor liver transplant (LDLT) compared to dead donor liver transplant (DDLT) recipients, socio-cultural, diet and lifestyle and varied immunosuppressive agents used may have confounded our analyses. We were, however, unable to exclude or control for all these factors in our analyses. A list of risk factors, both modifiable and non-modifiable, involved in NODAT development have been compiled in Supplementary Material 2. LDLT recipients have been noted to have lower incidences and lowered risk for NODAT compared to DDLT recipients [11,14,36]. Dif- 
ferences in the type of liver donors have been noted between various countries, which could affect the incidence of NODAT [59]. Another reason may be attributed to the varied diabetogenic effect of immunosuppressive agents and greater risk of diabetes due to cultural and lifestyle differences [9]. There is also variance in the immunosuppressive strategies and protocols in the different countries, with some using steroid-free regimens [60], and mTOR-I (mammalian target of rapamycin inhibitors) [12,61,62], which affects the incidence of NODAT. Lastly, variations in the indications for liver transplant were noted between countries, and this could have affected the incidence of NODAT. Asians were noted to be more commonly indicated for liver transplant due to hepatitis B virus (HBV), and HCC while patients from Western populations were more likely to need liver transplant due to $\mathrm{HCV}$ and alcoholic liver disease related liver failures [63].

Considering the high incidence of NODAT, there has been increasing calls for more stringent surveillance and management of NODAT. The International Consensus Meeting on Post-transplantation Diabetes Mellitus in 2014 suggested that besides the increased screening recommendations in the first year post-transplant, and annual screens thereafter, an additional pre-transplant baseline evaluation should be done to evaluate the risk of developing NODAT $[9,64]$. This includes a complete medical, family, and glucose history, as well as additional factors such as body weight and HCV status [9,64]. Individualisation of immunosuppressive treatment, to better balance the individual risks between transplant rejection and increasing their risk of developing NODAT, can also be implemented for patients to maximise their benefits from the treatment [9]. Additionally, screening for NODAT using postprandial glycemia and (glycated haemoglobin) $\mathrm{HbA} 1 \mathrm{c}$ are also recommended to better streamline investigations for NODAT [64]. A stepwise approach for the management of NODAT is also recommended, where guidance for lifestyle modifications and exercise to reduce the risk of developing NODAT after liver transplantation, followed by additional opinion-based guidance for pharmacological therapy [64]. These recommendations would allow for early identification and investigations of high-risk individuals for developing NODAT, reducing the morbidity and mortality of NODAT through early intervention [64].

\section{Strength and Limitations}

To the best of our knowledge, this is the most detailed meta-analysis analysing the incidence of NODAT after liver transplantation in various timepoints. Previous studies systematically reported the prevalence of NODAT [18], post-transplant metabolic diseases [65], compared the incidence of NODAT without considering the specific timepoints or were conducted before the introduction of the WHO and ADA guidelines [12,19]. Additionally, this meta-analysis compared the possible difference in Asian and European incidence of NODAT and suggests further analysis to confirm these findings. However, there are several limitations in this meta-analysis. Firstly, only English articles were included in this paper, which may limit the generalisability of the incidence of NODAT. Secondly, there were few articles examining the incidence of NODAT aside from the one-year timepoint (Table 2), and this may have led to a lack of generalisability of our findings and possible biasness of the results found. Additionally, we were unable to analyse the differences in steroid dosages due to tapered immunosuppressive regimen, which is individualised to each patient. Next, significant funnel plot asymmetry was found, and many studies were not located within the funnel plot. This which suggests that there is the presence of publication bias or heterogeneity in the current literature pool, which would have affected the results of the analysis. Lastly, most of the pooled estimates had a high $\mathrm{I}^{2}$ value $(>75 \%)$, suggesting that there is a large heterogeneity in our summary estimates. However, the use of $\mathrm{I}^{2}$ for assessing statistical heterogeneity in studies with large sample sizes, such as prevalence or incidence meta-analyses, is debatable, and previous studies have shown that overreliance on $\mathrm{I}^{2}$ to assess heterogeneity may be misleading $[26,66]$. 


\section{Conclusions}

In summary, this meta-analysis shows that NODAT is common after liver transplant with one quarter of individuals developing NODAT 10 years after transplant. The incidence of NODAT increases with years after liver transplantation, suggesting there are potentially reversible factors that could exacerbate NODAT. Further studies exploring the impact of ethnicity and genetics on the incidence of NODAT, and clinical trials examining specific pharmacotherapies for NODAT in liver transplant patients are also needed.

Supplementary Materials: The following are available online at https:/ /www.mdpi.com/2077-038 3/10/5/1045/s1; Supplementary Material 1: Search Strategy, Supplementary Material 2: Modifiable and non-modifiable factors implicated in NODAT, Supplementary Material 3: Funnel plot of incidence of NODAT at 6-months after Liver Transplantation, Supplementary Material 4: Funnel plot of incidence of NODAT at 3-years after Liver Transplantation, Supplementary Material 5: Funnel plot of incidence of NODAT at 5-years after Liver Transplantation.

Author Contributions: Study conception, Y.H.C. and C.H.N.; methodology, H.Q.M.T. and Y.H.C.; formal analysis Y.H.C., H.Q.M.T., C.H.N., D.J.H.T., S.Y.L.; data interpretation Y.H.C., H.Q.M.T., D.J.H.T., S.Y.L., D.Q.H., C.M.K. and M.D.M.; writing of first draft of paper, Y.H.C. and H.Q.M.T.; critical revision of article and final approval, Y.H.C., H.Q.M.T., C.H.N., D.J.H.T., S.Y.L., D.Q.H., C.M.K., M.D.M. All authors have read and agreed to the published version of the manuscript.

Funding: This research received no external funding.

Data Availability Statement: The data and articles presented in this study are openly available in Medline and Embase.

Conflicts of Interest: The authors declare no conflict of interest.

\section{References}

1. Schuppan, D.; Afdhal, N.H. Liver cirrhosis. Lancet 2008, 371, 838-851. [CrossRef]

2. Marchetti, P. New-onset diabetes after liver transplantation: From pathogenesis to management. Liver Transpl. 2005, 11, 612-620. [CrossRef]

3. Watt, K.D.; Pedersen, R.A.; Kremers, W.K.; Heimbach, J.K.; Charlton, M.R. Evolution of causes and risk factors for mortality post-liver transplant: Results of the NIDDK long-term follow-up study. Am. J. Transpl. 2010, 10, 1420-1427. [CrossRef] [PubMed]

4. Roccaro, G.A.; Goldberg, D.S.; Hwang, W.T.; Judy, R.; Thomasson, A.; Kimmel, S.E.; Forde, K.A.; Lewis, J.D.; Yang, Y.X. Sustained posttransplantation diabetes is associated with long-term major cardiovascular events following liver transplantation. Am. J. Transpl. 2018, 18, 207-215. [CrossRef] [PubMed]

5. Lv, C.; Zhang, Y.; Chen, X.; Huang, X.; Xue, M.; Sun, Q.; Wang, T.; Liang, J.; He, S.; Gao, J.; et al. New-onset diabetes after liver transplantation and its impact on complications and patient survival. J. Diabetes 2015, 7, 881-890. [CrossRef] [PubMed]

6. Ling, Q.; Xu, X.; Xie, H.; Wang, K.; Xiang, P.; Zhuang, R.; Shen, T.; Wu, J.; Wang, W.; Zheng, S. New-onset diabetes after liver transplantation: A national report from China Liver Transplant Registry. Liver Int. 2016, 36, 705-712. [CrossRef] [PubMed]

7. Navasa, M.; Bustamante, J.; Marroni, C.; González, E.; Andreu, H.; Esmatjes, E.; García-Valdecasas, J.C.; Grande, L.; Cirera, I.; Rimola, A.; et al. Diabetes mellitus after liver transplantation: Prevalence and predictive factors. J. Hepatol. 1996, $25,64-71$. [CrossRef]

8. Moon, J.I.; Barbeito, R.; Faradji, R.N.; Gaynor, J.J.; Tzakis, A.G. Negative impact of new-onset diabetes mellitus on patient and graft survival after liver transplantation: Long-term follow up. Transplantation 2006, 82, 1625-1628. [CrossRef]

9. Davidson, J.; Wilkinson, A.; Dantal, J.; Dotta, F.; Haller, H.; Hernandez, D.; Kasiske, B.L.; Kiberd, B.; Krentz, A.; Legendre, C.; et al. New-onset diabetes after transplantation: 2003 International consensus guidelines. Proceedings of an international expert panel meeting. Barcelona, Spain, 19 February 2003. Transplantation 2003, 75, SS3-S24.

10. Pham, P.-T.T.; Pham, P.-M.T.; Pham, S.V.; Pham, P.-A.T.; Pham, P.-C.T. New onset diabetes after transplantation (NODAT): An overview. Diabetes Metab. Syndr. Obes. 2011, 4, 175-186. [CrossRef]

11. Kuo, H.T.; Sampaio, M.S.; Ye, X.; Reddy, P.; Martin, P.; Bunnapradist, S. Risk factors for new-onset diabetes mellitus in adult liver transplant recipients, an analysis of the Organ Procurement and Transplant Network/United Network for Organ Sharing database. Transplantation 2010, 89, 1134-1140. [CrossRef] [PubMed]

12. Li, D.-W.; Lu, T.-F.; Hua, X.-W.; Dai, H.-J.; Cui, X.-L.; Zhang, J.-J.; Xia, Q. Risk factors for new onset diabetes mellitus after liver transplantation: A meta-analysis. World J. Gastroenterol. 2015, 21, 6329-6340. [CrossRef]

13. Li, Z.; Sun, F.; Hu, Z.; Xiang, J.; Zhou, J.; Yan, S.; Wu, J.; Zhou, L.; Zheng, S. New-onset diabetes mellitus in liver transplant recipients with hepatitis C: Analysis of the national database. Transpl. Proc. 2016, 48, 138-144. [CrossRef] [PubMed] 
14. Yadav, A.D.; Chang, Y.-H.; Aqel, B.A.; Byrne, T.J.; Chakkera, H.A.; Douglas, D.D.; Mulligan, D.C.; Rakela, J.; Vargas, H.E.; Carey, E.J. New onset diabetes mellitus in living donor versus deceased donor liver transplant recipients: Analysis of the UNOS/OPTN database. J. Transpl. 2013, 2013, 269096. [CrossRef] [PubMed]

15. Xue, M.; Lv, C.; Chen, X.; Liang, J.; Zhao, C.; Zhang, Y.; Huang, X.; Sun, Q.; Wang, T.; Gao, J.; et al. Donor liver steatosis: A risk factor for early new-onset diabetes after liver transplantation. J. Diabetes Investig. 2017, 8, 181-187. [CrossRef]

16. Kelava, T.; Turcic, P.; Markotic, A.; Ostojic, A.; Sisl, D.; Mrzljak, A. Importance of genetic polymorphisms in liver transplantation outcomes. World J. Gastroenterol. 2020, 26, 1273-1285. [CrossRef] [PubMed]

17. Sulanc, E.; Lane, J.T.; Puumala, S.E.; Groggel, G.C.; Wrenshall, L.E.; Stevens, R.B. New-onset diabetes after kidney transplantation: An application of 2003 International Guidelines. Transplantation 2005, 80, 945-952. [CrossRef] [PubMed]

18. Peláez-Jaramillo, M.J.; Cárdenas-Mojica, A.A.; Gaete, P.V.; Mendivil, C.O. Post-liver transplantation diabetes mellitus: A review of relevance and approach to treatment. Diabetes Ther. 2018, 9, 521-543. [CrossRef] [PubMed]

19. Heisel, O.; Heisel, R.; Balshaw, R.; Keown, P. New onset diabetes mellitus in patients receiving calcineurin inhibitors: A systematic review and meta-analysis. Am. J. Transpl. 2004, 4, 583-595. [CrossRef]

20. Stroup, D.F.; Berlin, J.A.; Morton, S.C.; Olkin, I.; Williamson, G.D.; Rennie, D.; Moher, D.; Becker, B.J.; Sipe, T.A.; Thacker, S.B. Meta-analysis of observational studies in epidemiology: A proposal for reporting. Meta-analysis Of Observational Studies in Epidemiology (MOOSE) group. JAMA 2000, 283, 2008-2012. [CrossRef]

21. Moher, D.; Liberati, A.; Tetzlaff, J.; Altman, D.G.; Group, P. Preferred reporting items for systematic reviews and meta-analyses: The PRISMA statement. PLoS Med. 2009, 6, e1000097. [CrossRef]

22. Wan, X.; Wang, W.; Liu, J.; Tong, T. Estimating the sample mean and standard deviation from the sample size, median, range and/or interquartile range. BMC Med. Res. Methodol. 2014, 14, 135. [CrossRef]

23. Schwarzer, G.; Chemaitelly, H.; Abu-Raddad, L.J.; Rücker, G. Seriously misleading results using inverse of Freeman-Tukey double arcsine transformation in meta-analysis of single proportions. Res. Synth. Methods 2019, 10, 476-483. [CrossRef]

24. Ye, Q.; Zou, B.; Yeo, Y.H.; Li, J.; Huang, D.Q.; Wu, Y.; Yang, H.; Liu, C.; Kam, L.Y.; Tan, X.X.E.; et al. Global prevalence, incidence, and outcomes of non-obese or lean non-alcoholic fatty liver disease: A systematic review and meta-analysis. Lancet Gastroenterol. Hepatol. 2020, 5, 739-752. [CrossRef]

25. Fairbrass, K.M.; Costantino, S.J.; Gracie, D.J.; Ford, A.C. Prevalence of irritable bowel syndrome-type symptoms in patients with inflammatory bowel disease in remission: A systematic review and meta-analysis. Lancet Gastroenterol. Hepatol. 2020, 5 , 1053-1062. [CrossRef]

26. Rücker, G.; Schwarzer, G.; Carpenter, J.R.; Schumacher, M. Undue reliance on I2 in assessing heterogeneity may mislead. BMC Med. Res. Methodol. 2008, 8, 79. [CrossRef] [PubMed]

27. Chin, Y.H.; Ng, C.H.; Lee, M.H.; Koh, J.W.H.; Kiew, J.; Yang, S.P.; Sundar, G.; Khoo, C.M. Prevalence of thyroid eye disease in Graves' disease: A meta-analysis and systematic review. Clin. Endocrinol. 2020, 93, 363-374. [CrossRef]

28. Koo, C.H.; Chang, J.H.E.; Syn, N.L.; Wee, I.J.Y.; Mathew, R. Systematic review and meta-analysis on colorectal cancer findings on colonic evaluation after CT-confirmed acute diverticulitis. Dis. Colon. Rectum. 2020, 63, 701-709. [CrossRef]

29. Sterne, J.A.C.; Sutton, A.J.; Ioannidis, J.P.A.; Terrin, N.; Jones, D.R.; Lau, J.; Carpenter, J.; Rücker, G.; Harbord, R.M.; Schmid, C.H.; et al. Recommendations for examining and interpreting funnel plot asymmetry in meta-analyses of randomised controlled trials. BMJ 2011, 343, d4002. [CrossRef] [PubMed]

30. Wells, G.; Shea, B.; O'Connell, D.; Peterson, j.; Welch, V.; Losos, M.; Tugwell, P. The Newcastle-Ottawa Scale (NOS) for Assessing the Quality of Non-Randomized Studies in Meta-Analysis; Ottawa Hospital Research Institute: Ottawa, ON, USA, 2000.

31. Jain, A.B.; Kashyap, R.; Rakela, J.; Starzl, T.E.; Fung, J.J. Primary adult liver transplantation under tacrolimus: More than 90 months actual follow-up survival and adverse events. Liver Transpl. Surg. 1999, 5, 144-150. [CrossRef]

32. Driscoll, C.J.; Cashion, A.K.; Hathaway, D.K.; Thompson, C.; Conley, Y.; Gaber, O.; Vera, S.; Shokouh-Amiri, H. Posttransplant diabetes mellitus in liver transplant recipients. Prog. Transpl. 2006, 16, 110-116. [CrossRef]

33. Lieber, S.R.; Lee, R.-A.; Jiang, Y.; Reuter, C.; Watkins, R.; Szempruch, K.; Gerber, D.A.; Desai, C.S.; DeCherney, G.S.; Barritt, A.S.T. The impact of post-transplant diabetes mellitus on liver transplant outcomes. Clin. Transplant. 2019, 33, e13554. [CrossRef]

34. Younossi, Z.; Stepanova, M.; Saab, S.; Trimble, G.; Mishra, A.; Henry, L. The association of hepatitis C virus infection and post-liver transplant diabetes: Data from 17000 HCV-infected transplant recipients. Aliment. Pharmacol. Ther. 2015, 41, 209-217. [CrossRef] [PubMed]

35. Stepanova, M.; Henry, L.; Garg, R.; Kalwaney, S.; Saab, S.; Younossi, Z. Risk of de novo post-transplant type 2 diabetes in patients undergoing liver transplant for non-alcoholic steatohepatitis. BMC Gastroenterol. 2015, 15, 175. [CrossRef] [PubMed]

36. Carey, E.J.; Aqel, B.A.; Byrne, T.J.; Douglas, D.D.; Rakela, J.; Vargas, H.E.; Moss, A.A.; Mulligan, D.C.; Reddy, K.S.; Chakkera, H.A. Pretransplant fasting glucose predicts new-onset diabetes after liver transplantation. J. Transpl. 2012, 2012, 614781. [CrossRef]

37. Song, J.-L.; Gao, W.; Zhong, Y.; Yan, L.-N.; Yang, J.-Y.; Wen, T.-F.; Li, B.; Wang, W.-T.; Wu, H.; Xu, M.-Q.; et al. Minimizing tacrolimus decreases the risk of new-onset diabetes mellitus after liver transplantation. World J. Gastroenterol. 2016, 22, $2133-2141$. [CrossRef]

38. Zhao, J.; Yan, L.; Li, B.; Zeng, Y.; Wen, T.; Zhao, J.; Wang, W.; Xu, M.; Yang, J.; Ma, Y.; et al. Diabetes mellitus after living donor liver transplantation: Data from mainland China. Transpl. Proc. 2009, 41, 1756-1760. [CrossRef] 
39. Cen, C.; Fang, H.-X.; Yu, S.-F.; Liu, J.-M.; Liu, Y.-X.; Zhou, L.; Yu, J.; Zheng, S.-S. Association between ADIPOQ gene polymorphisms and the risk of new-onset diabetes mellitus after liver transplantation. Hepatobiliary Pancreat. Dis. Int. 2017, 16, 602-609. [CrossRef]

40. Saliba, F.; Rostaing, L.; Gugenheim, J.; Durand, F.; Radenne, S.; Leroy, V.; Neau-Cransac, M.; Calmus, Y.; Salame, E.; Pageaux, G.-P.; et al. Corticosteroid-sparing and optimization of mycophenolic acid exposure in liver transplant recipients receiving mycophenolate mofetil and tacrolimus: A randomized, multicenter study. Transplantation 2016, 100, 1705-1713. [CrossRef]

41. Oufroukhi, L.; Kamar, N.; Muscari, F.; Lavayssiere, L.; Guitard, J.; Ribes, D.; Esposito, L.; Alric, L.; Hanaire, H.; Rostaing, L. Predictive factors for posttransplant diabetes mellitus within one-year of liver transplantation. Transplantation 2008, 85, $1436-1442$. [CrossRef]

42. Saliba, F.; Lakehal, M.; Pageaux, G.P.; Roche, B.; Vanlemmens, C.; Duvoux, C.; Dumortier, J.; Salamé, E.; Calmus, Y.; Maugendre, D. Risk factors for new-onset diabetes mellitus following liver transplantation and impact of hepatitis $\mathrm{C}$ infection: An observational multicenter study. Liver Transpl. 2007, 13, 136-144. [CrossRef]

43. Oommen, T.; Arun, C.S.; Kumar, H.; Nair, V.; Jayakumar, R.V.; Sudhindran, S.; Praveen, V.P.; Abraham, N.B.N.; Menon, U. Incidence of new-onset diabetes and posttransplant metabolic syndrome after liver transplantation-A prospective study from South India. Indian J. Endocrinol. Metab. 2020, 24, 165-169. [CrossRef]

44. Varghese, J.; Reddy, M.S.; Venugopal, K.; Perumalla, R.; Narasimhan, G.; Arikichenin, O.; Shanmugam, V.; Shanmugam, N.; Srinivasan, V.; Jayanthi, V.; et al. Tacrolimus-related adverse effects in liver transplant recipients: Its association with trough concentrations. Indian J. Gastroenterol. 2014, 33, 219-225. [CrossRef] [PubMed]

45. Honda, M.; Asonuma, K.; Hayashida, S.; Suda, H.; Ohya, Y.; Lee, K.-J.; Yamamoto, H.; Takeichi, T.; Inomata, Y. Incidence and risk factors for new-onset diabetes in living-donor liver transplant recipients. Clin. Transplant. 2013, 27, 426-435. [CrossRef]

46. Yagi, S.; Kaido, T.; Iida, T.; Yoshizawa, A.; Okajima, H.; Uemoto, S. New-onset diabetes mellitus after living-donor liver transplantation: Association with graft synthetic function. Surg. Today 2017, 47, 733-742. [CrossRef] [PubMed]

47. Cuervas-Mons, V.; Herrero, J.I.; Gomez, M.A.; Gonzalez-Pinto, I.; Serrano, T.; de la Mata, M.; Fabregat, J.; Gastaca, M.; Bilbao, I.; Varo, E.; et al. Impact of tacrolimus and mycophenolate mofetil regimen vs. a conventional therapy with steroids on cardiovascular risk in liver transplant patients. Clin. Transpl. 2015, 29, 667-677. [CrossRef]

48. Rubin, A.; Sanchez-Montes, C.; Aguilera, V.; Juan, F.S.; Ferrer, I.; Moya, A.; Montalva, E.; Pareja, E.; Lopez-Andujar, R.; Prieto, M.; et al. Long-term outcome of 'long-term liver transplant survivors'. Transpl. Int. 2013, 26, 740-750. [CrossRef] [PubMed]

49. Aravinthan, A.D.; Fateen, W.; Doyle, A.C.; Venkatachalapathy, S.V.; Issachar, A.; Galvin, Z.; Sapisochin, G.; Cattral, M.S.; Ghanekar, A.; McGilvray, I.D.; et al. The impact of preexisting and post-transplant diabetes mellitus on outcomes following liver transplantation. Transplantation 2019, 103, 2523-2530. [CrossRef] [PubMed]

50. Hashim, M.S.; Alsabaawy, M.; Afify, S.; El-Azab, G.; Omar, N. Incidence and risk factors for diabetes, hypertension and hyperlipidemia after liver transplantation. J. Gastroenterol. Hepatol. Res. 2020, 9, 3077-3081. [CrossRef]

51. Gebhardt, S.; Jara, M.; Malinowski, M.; Seehofer, D.; Puhl, G.; Pratschke, J.; Stockmann, M. Risk factors of metabolic disorders after liver transplantation: An analysis of data from fasted patients. Transplantation 2015, 99, 1243-1249. [CrossRef] [PubMed]

52. Parvizi, Z.; Azarpira, N.; Kohan, L.; Darai, M.; Kazemi, K.; Parvizi, M.M. Association between E23K variant in KCNJ11 gene and new-onset diabetes after liver transplantation. Mol. Biol. Rep. 2014, 41, 6063-6069. [CrossRef]

53. Cho, Y.; Lee, M.J.; Choe, E.Y.; Jung, C.H.; Joo, D.J.; Kim, M.S.; Cha, B.S.; Park, J.-Y.; Kang, E.S. Statin therapy is associated with the development of new-onset diabetes after transplantation in liver recipients with high fasting plasma glucose levels. Liver Transpl. 2014, 20, 557-563. [CrossRef] [PubMed]

54. Liu, F.-C.; Lin, J.-R.; Chen, H.-P.; Tsai, Y.-F.; Yu, H.-P. Prevalence, predictive factors, and survival outcome of new-onset diabetes after liver transplantation: A population-based cohort study. Medicine 2016, 95, e3829. [CrossRef]

55. Hartog, H.; May, C.J.H.; Corbett, C.; Phillips, A.; Tomlinson, J.W.; Mergental, H.; Isaac, J.; Bramhall, S.; Mirza, D.F.; Muiesan, P.; et al. Early occurrence of new-onset diabetes after transplantation is related to type of liver graft and warm ischaemic injury. Liver Int. 2015, 35, 1739-1747. [CrossRef] [PubMed]

56. John, P.R.; Thuluvath, P.J. Outcome of patients with new-onset diabetes mellitus after liver transplantation compared with those without diabetes mellitus. Liver Transpl. 2002, 8, 708-713. [CrossRef] [PubMed]

57. Wilkinson, A.; Davidson, J.; Dotta, F.; Home, P.D.; Keown, P.; Kiberd, B.; Jardine, A.; Levitt, N.; Marchetti, P.; Markell, M.; et al. Guidelines for the treatment and management of new-onset diabetes after transplantation. Clin. Transpl. 2005, 19, 291-298. [CrossRef]

58. Jain, A.; Reyes, J.; Kashyap, R.; Dodson, SF.; Demetris, AJ.; Ruppert, K.; Abu-Elmagd, K.; Marsh, W.; Madariaga, J.; Mazariegos, G.; et al. Long-term survival after liver transplantation in 4000 consecutive patients at a single center. Ann. Surg. 2000, 23, 490-500. [CrossRef] [PubMed]

59. Tanaka, K.; Ogura, Y.; Kiuchi, T.; Inomata, Y.; Uemoto, S.; Furukawa, H. Living donor liver transplantation: Eastern experiences. HPB (Oxf.) 2004, 6, 88-94. [CrossRef] [PubMed]

60. Sgourakis, G.; Radtke, A.; Fouzas, I.; Mylona, S.; Goumas, K.; Gockel, I.; Lang, H.; Karaliotas, C. Corticosteroid-free immunosuppression in liver transplantation: A meta-analysis and meta-regression of outcomes. Transpl. Int. 2009, 22, 892-905. [CrossRef] [PubMed]

61. Klintmalm, G.B.; Nashan, B. The Role of mTOR inhibitors in liver transplantation: Reviewing the evidence. J. Transpl. 2014, 2014, 845438. [CrossRef] 
62. Tan, P.S.; Muthiah, M.D.; Koh, T.; Teoh, Y.L.; Chan, A.; Kow, A.; Zheng, Q.; Kwon, C.H.D.; Lee, G.H.; Lesmana, C.R.A.; et al. Asian liver transplant network clinical guidelines on immunosuppression in liver transplantation. Transplantation 2019, 103, 470-480. [CrossRef] [PubMed]

63. Shukla, A.; Vadeyar, H.; Rela, M.; Shah, S. Liver transplantation: East versus West. J. Clin. Exp. Hepatol. 2013, 3, $243-253$. [CrossRef] [PubMed]

64. Sharif, A.; Hecking, M.; de Vries, A.P.J.; Porrini, E.; Hornum, M.; Rasoul-Rockenschaub, S.; Berlakovich, G.; Krebs, M.; KautzkyWiller, A.; Schernthaner, G.; et al. Proceedings from an international consensus meeting on posttransplantation diabetes mellitus: Recommendations and future directions. Am. J. Transpl. 2014, 14, 1992-2000. [CrossRef] [PubMed]

65. Thoefner, L.B.; Rostved, A.A.; Pommergaard, H.C.; Rasmussen, A. Risk factors for metabolic syndrome after liver transplantation A systematic review and meta-analysis. Transpl. Rev. 2018, 32, 69-77. [CrossRef] [PubMed]

66. Borges Migliavaca, C.; Stein, C.; Colpani, V.; Barker, T.H.; Munn, Z.; Falavigna, M. How are systematic reviews of prevalence conducted? A methodological study. BMC Med. Res. Methodol. 2020, 20, 96. [CrossRef] [PubMed] 